\title{
XMM-Newton observations of the Soft Gamma Ray Repeater SGR 1627-41 in a low luminosity state
}

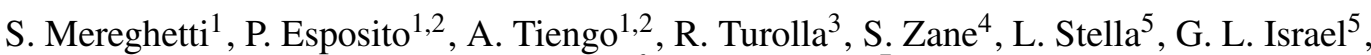 \\ M. Feroci ${ }^{6}$, and A. Treves
}

\footnotetext{
1 INAF - Istituto di Astrofisica Spaziale e Fisica Cosmica Milano, via Bassini 15, 20133 Milano, Italy e-mail: sandro@iasf-milano.inaf.it

2 Università degli Studi di Milano, Dipartimento di Fisica, via Celoria 16, 20133 Milano, Italy

3 Università di Padova, Dipartimento di Fisica, via Marzolo 8, 35131 Padova, Italy

4 Mullard Space Science Laboratory, University College London, Holmbury St. Mary, Dorking Surrey, RH5 6NT, UK

5 INAF - Osservatorio Astronomico di Roma, via Frascati 33, 00040 Monteporzio Catone, Roma, Italy

6 INAF - Istituto di Astrofisica Spaziale e Fisica Cosmica Roma, via Fosso del Cavaliere 100, 00133 Roma, Italy

7 Università degli Studi dell'Insubria, via Valleggio 11, 22100 Como, Italy
}

Received 15 September 2005 / Accepted 11 January 2006

\section{ABSTRACT}

The sky region containing the soft gamma-ray repeater SGR 1627-41 has been observed three times with XMM-Newton in February and September 2004. SGR 1627-41 has been detected with an absorbed flux of $\sim 9 \times 10^{-14} \mathrm{erg} \mathrm{cm}^{-2} \mathrm{~s}^{-1}(2-10 \mathrm{keV})$. For a distance of $11 \mathrm{kpc}$, this corresponds to a luminosity of $\sim 3 \times 10^{33} \mathrm{erg} \mathrm{s}^{-1}$, the smallest ever observed for a Soft Gamma Repeater and possibly related to the long period of inactivity of this source. The observed flux is smaller than that seen with Chandra in 2001-2003, suggesting that the source was still fading and had not yet reached a steady quiescent level. The spectrum is equally well fit by a steep power law (photon index $\sim 3.2$ ) or by a blackbody with temperature $k T \sim 0.8 \mathrm{keV}$. We also report on the INTEGRAL transient IGR J16358-4726 that lies at $\sim 10^{\prime}$ from SGR 1627-41. It was detected only in September 2004 with a luminosity of $\sim 4 \times 10^{33} \mathrm{erg} \mathrm{s}^{-1}$ (for $d=7 \mathrm{kpc}$ ), while in February 2004 it was at least a factor 10 fainter.

Key words. stars: individual: SGR 1627-41 - X-rays: individuals: IGR J16358-4726 - stars: neutron

\section{Introduction}

SGR 1627-41 is one of the four confirmed Soft Gamma-ray Repeaters (SGRs) that are currently known. According to the widely accepted magnetar model (Duncan \& Thompson 1992; Thompson \& Duncan 1995), these sources are isolated neutron stars in which the high-energy emission is powered by ultra-strong magnetic fields $\left(B \sim 10^{14}-10^{15} \mathrm{G}\right)$. The distinctive characteristic of SGRs is the emission, during sporadic periods of activity, of short bursts $(<1 \mathrm{~s})$ of hard X-rays with super-Eddington peak luminosity $L \sim 10^{40}-10^{41} \mathrm{erg} \mathrm{s}^{-1}$. Persistent (i.e. non-bursting) emission is also observed from SGRs in the soft X-ray range $(<10 \mathrm{keV})$, with typical luminosity of $\sim 10^{35} \mathrm{erg} \mathrm{s}^{-1}$. Periodic pulsations at several seconds, reflecting the neutron star rotation, are observed in three SGRs. Occasionally, SGRs also emit energetic (giant) flares with luminosity from $\sim 10^{43} \mathrm{erg} \mathrm{s}^{-1}$ up to $10^{47} \mathrm{erg} \mathrm{s}^{-1}$. For a review of the properties of these sources see Woods \& Thompson (2004).

It is interesting to study the relation between the properties of the persistent X-ray emission and the level of SGR bursting and flaring activity. In fact the persistent soft X-rays are thought to consist, at least in part, of thermal emission from the neutron star surface, whose properties, e.g. temperature and magnetization, can be influenced by the largely non-thermal phenomena responsible for the bursts.

The 1-10 keV luminosity of the two SGRs which have been more active in recent years, SGR 1806-20 and SGR 1900+14, displayed only moderate (factor $\sim 2$ ) long term variations around average values of $\sim 5 \times 10^{35} d_{15}^{2} \mathrm{erg} \mathrm{s}^{-1}$ and $\sim 1 \times 10^{35} d_{10}^{2}$ erg s$^{-1}$ respectively ${ }^{1}$ (e.g., Mereghetti et al. 2005; Woods et al. 2001). The SGR in the Large Magellanic Cloud, SGR 0526-66, has a similar luminosity of $\sim 10^{36} d_{55}^{2} \mathrm{erg} \mathrm{s}^{-1}$ (Kulkarni et al. 2003), despite no bursts have been detected from this source since $1984^{2}$. The only difference with respect to the two more active SGRs mentioned above is that its spectrum is much softer, requiring a power law photon index larger than $\sim 3$ (Kulkarni et al. 2003).

SGR 1627-41 was discovered in 1998, when more than 100 bursts were observed with different satellites (CGRO, Woods et al. 1999; Ulysses, Hurley et al. 1999; Wind, Mazets et al. 1999; RXTE, Smith et al. 1999; BeppoSAX,

\footnotetext{
${ }^{1}$ We indicate with $d_{N}$ the distance in units of $N \mathrm{kpc}$.

2 Some bursts might have been missed in the time interval 19851991 due to the lack of suitable detectors in operation (see Woods \& Thompson 2004).
} 
Table 1. Spectral results on SGR 1627-41. The errors of the BeppoSAX and XMM-Newton values are at $1 \sigma$ for a single parameter of interest, and, for the fluxes, take into account both the statistical and spectral uncertainties. The ASCA and Chandra values are from Kouveliotou et al. (2003).

\begin{tabular}{lccccc}
\hline \hline Satellite & Observation date & $\begin{array}{c}N_{\mathrm{H}} \\
10^{22} \mathrm{~cm}^{-2}\end{array}$ & $\begin{array}{c}\text { photon } \\
\text { index }\end{array}$ & $\begin{array}{c}\text { Observed flux } \\
10^{-12} \mathrm{erg} \mathrm{cm}^{-2} \mathrm{~s}^{-1}\end{array}$ & $\begin{array}{c}\text { Unabsorbed flux } \\
10^{-12} \mathrm{erg} \mathrm{cm}^{-2} \mathrm{~s}^{-1}\end{array}$ \\
\hline BeppoSAX & 1998 August 6-7 & $10 \pm 1$ & $2.6 \pm 0.3$ & $2.5 \pm 0.2$ & $5.9_{-1.0}^{+1.4}$ \\
BeppoSAX & 1998 September 16 & $10 \pm 2$ & $2.8 \pm 0.4$ & $1.9 \pm 0.2$ & $4.6_{-0.9}^{+1.3}$ \\
BeppoSAX & 1999 August 8-10 & $5 \pm 2$ & $1.9_{-0.7}^{+0.4}$ & $0.7 \pm 0.1$ & $1.1 \pm 0.3$ \\
BeppoSAX & 2000 September 5-7 & $8_{-4}^{+5}$ & $3.0_{-0.9}^{+1.3}$ & $0.4_{-0.1}^{+0.2}$ & $1.1 \pm 0.4$ \\
XMM-Newton & 2004 September 22 & $11.4_{-1.9}^{+4.3}$ & $3.7 \pm 0.5$ & $0.09 \pm 0.02$ & $0.32 \pm 0.09$ \\
\hline BeppoSAX & 1998 August 6-7 & $9^{(b)}$ & $2.4 \pm 0.1$ & $2.6 \pm 0.1$ & $5.2 \pm 0.3$ \\
BeppoSAX & 1998 September 16 & $9^{(b)}$ & $2.6 \pm 0.2$ & $1.9 \pm 0.1$ & $4.2 \pm 0.3$ \\
ASCA & 1999 February 26-28 & $9^{(b)}$ & $3.24 \pm 0.24$ & $1.07 \pm 0.16$ & $2.76 \pm 0.41$ \\
BeppoSAX & 1999 August 8-10 & $9^{(b)}$ & $2.5 \pm 0.3$ & $0.7 \pm 0.1$ & $1.5 \pm 0.2$ \\
BeppoSAX & 2000 September 5-7 & $9^{(b)}$ & $3.3 \pm 0.5$ & $0.4 \pm 0.1$ & $1.1 \pm 0.2$ \\
Chandra & 2001 September 30 & $9^{(b)}$ & $2.17 \pm 0.30$ & $0.14 \pm 0.02$ & $0.267 \pm 0.04$ \\
Chandra & 2002 August 19 & $9^{(b)}$ & $2.95 \pm 0.36$ & $0.11 \pm 0.02$ & $0.266 \pm 0.04$ \\
XMM-Newton & 2004 February 16 & $9^{(b)}$ & $3.2^{(b)}$ & $0.12 \pm 0.02$ & $0.32 \pm 0.06$ \\
XMM-Newton & 2004 September 4 & $9^{(b)}$ & $3.2^{(b)}$ & $0.10 \pm 0.02$ & $0.26 \pm 0.04$ \\
XMM-Newton & 2004 September 22 & $9^{(b)}$ & $3.2 \pm 0.3$ & $0.09 \pm 0.01$ & $0.23 \pm 0.04$ \\
\hline
\end{tabular}

${ }^{a}$ in the $2-10 \mathrm{keV}$ energy range.

${ }^{b}$ fixed value.

Feroci et al. 1998). No other bursts from this source have been reported to date. Its soft X-ray counterpart was identified with BeppoSAX in 1998 at a luminosity level of $\sim 10^{35} d_{11}^{2} \mathrm{erg} \mathrm{s}^{-1}$ (Woods et al. 1999). Subsequent observations, carried out over a time span of five years with BeppoSAX, ASCA and Chandra, showed a monotonic decrease in the luminosity, interpreted as evidence for cooling of the neutron star surface after the deep crustal heating that occurred during the 1998 period of SGR activity (Kouveliotou et al. 2003). The latest Chandra observation (March 2003) yielded an X-ray flux consistent with that measured in September 1999, suggesting that the luminosity of SGR 1627-41 settled at its "quiescent" level of $\sim 4 \times 10^{33} d_{11}^{2} \mathrm{erg} \mathrm{s}^{-1}$. To further study the luminosity evolution of SGR 1627-41 we observed it with XMM-Newton in September 2004. The SGR was also serendipitously detected in two other XMM-Newton observations pointed on the transient IGR J16358-4726 (Patel et al. 2004), which lies at an angular distance of $\sim 10^{\prime}$. We report here also the results of these serendipitous detections, as well as a reanalysis of the BeppoSAX observations. For completeness, we report also the results of the three XMM-Newton observations for IGR J16358-4726.

\section{Data analysis and results}

\subsection{SGR 1627-41: on-axis data}

Our observation of SGR 1627-41 was carried out on 2004 September 22 and lasted about 52 ks. Here we report on the results obtained with the three EPIC CCD cameras (Turner et al. 2001; Strüder et al. 2001) since the target was too faint for the RGS instruments. All the CCDs were operated in Small Window mode, yielding time resolution of $6 \mathrm{~ms}(\mathrm{PN})$ and $0.3 \mathrm{~s}$ (MOS), and the medium thickness filter was used.

A faint, but statistically significant, source was detected at the coordinates of SGR 1627-41 (Wachter et al. 2004) in all the EPIC cameras. Its background-subtracted count rates were $2.5 \pm 0.4$ and $3.0 \pm 0.4$ counts ks${ }^{-1}$ in the two MOS cameras (with net exposure times of $50.3 \mathrm{ks}$ each) and $9.4 \pm$ 1.2 counts ks $^{-1}$ in the PN (net exposure $36.2 \mathrm{ks}$ ). We extracted the source spectra using a circular extraction region with $25^{\prime \prime}$ radius and the background from source free regions of the same observation. The spectra were rebinned to have at least 30 counts per channel. The spectra from the three cameras were fitted together, with the appropriate response matrices, to simple models. Although the small number of counts allowed us to carry out only a limited spectral analysis, there is clear evidence for a rather soft spectrum. Both a steep power law (photon index $\Gamma=3.7 \pm 0.5$ ) and a blackbody with temperature $k T_{\mathrm{BB}}=0.8_{-0.1}^{+0.2} \mathrm{keV}$ gave acceptable fits. The best fit power-law parameters are given in Table 1, where for comparison with previous work, we also report the values obtained by keeping the absorption fixed at the average value of the other observations, $N_{\mathrm{H}}=9 \times 10^{22} \mathrm{~cm}^{-2}$ (Kouveliotou et al. 2003). The blackbody fit gives an absorption $N_{\mathrm{H}}=\left(6.8_{-2.3}^{+2.7}\right) \times 10^{22} \mathrm{~cm}^{-2}$, an observed 2-10 keV flux of $(7.5 \pm 1.5) \times 10^{-14} \mathrm{erg} \mathrm{cm}^{-2} \mathrm{~s}^{-1}$, and, for an assumed distance of $11 \mathrm{kpc}$, an emitting radius of $0.2 \pm 0.1 \mathrm{~km}$.

We searched for periodic pulsations, using a small extraction region in order to reduce the fraction of background counts ( $45 \%$ of the total counts within the adopted $10^{\prime \prime}$ radius circle). The pulsations were not detected, but due to the limited statistics the upper limits on the source pulsed fraction are not particularly constraining. No evidence for bursts or other flux variations was seen.

\subsection{SGR 1627-41: off-axis data}

Two XMM-Newton observations of IGR J16358-4726 were carried out on 2004 February 16 and September 4, with 
a duration of about $30 \mathrm{ks}$ each. In both observations SGR 1627-41 was detected with EPIC at an off-axis angle of $9.6^{\prime}$, where, owing to the vignetting effect, the effective area is about half of the on-axis one. The background-subtracted PN count rates were $5.4 \pm 0.8$ and $4.2 \pm 0.6$ counts ks${ }^{-1}$, respectively. The counts statistics was too poor for a spectral analysis, but the source hardness ratios were consistent with the ones of the on-axis observation. We therefore computed the source fluxes given in Table 1 by fixing $N_{\mathrm{H}}=9 \times 10^{22} \mathrm{~cm}^{-2}$ and assuming the same photon index as the on-axis observation.

\subsection{IGR J16358-4726}

The transient IGR J16358-4726 was discovered in the hard X-ray band $(>15 \mathrm{keV})$ with INTEGRAL in March 2003 (Revnivtsev et al. 2003). Lying at a small angular distance from SGR 1627-41, this source was serendipitously present in several observations of the soft repeater. During the March-April 2003 outburst, periodic pulsations at 5880 s were discovered with Chandra (Patel et al. 2004). It is not clear yet whether they correspond to the spin period of a neutron star in a High Mass X-ray Binary or the orbital period of a Low Mass X-ray Binary.

IGR J16358-4726 was detected only in the two observations of September 2004. The best data were obtained on September 4 , when the source was detected on-axis, with background-subtracted count rates of $4.4 \pm 0.4,4.3 \pm 0.4$ and $18.9 \pm 1.0$ counts ks ${ }^{-1}$ in the MOS1, MOS2 and PN, respectively. The spectral analysis of these data, carried out as described in Sect. 2.1, yielded the following best fit parameters for an absorbed power law model: $\Gamma=1.5 \pm 0.5$, $N_{\mathrm{H}}=(20 \pm 5) \times 10^{22} \mathrm{~cm}^{-2}$, observed $2-10 \mathrm{keV}$ flux $=$ $(3.1 \pm 0.6) \times 10^{-13} \mathrm{erg} \mathrm{cm}^{-2} \mathrm{~s}^{-1}$. The background subtracted light curve folded at $5880 \mathrm{~s}$ (Fig. 1), shows that the periodic modulation, observed with Chandra when the source was a factor 200 more luminous, is present also in this lower intensity state.

In the subsequent observation (September 22) IGR J16358-4726 was detected only in the two MOS cameras, since its off-axis position was outside the region covered by the PN in Small Window mode. Although the limited statistics ( 200 net counts in total) do not allow us to see the pulsations or to significantly constrain the spectral parameters, the MOS count rates were consistent with the intensity and spectral shape measured 18 days earlier.

Adopting a power law spectrum with photon index $\Gamma=1.3$ and $N_{\mathrm{H}}=2 \times 10^{23} \mathrm{~cm}^{-2}$, in order to compare with the previous detections with ASCA and BeppoSAX (Patel et al. 2004), the September 2004 data correspond to an unabsorbed flux of $7.2 \times 10^{-13} \mathrm{erg} \mathrm{cm}^{-2} \mathrm{~s}^{-1}(2-10 \mathrm{keV})$. This is a factor five below that seen with the above satellites in 1999, and the smallest flux detected from IGR J16358-4726. For this spectrum, the February 2004 observation, yields a $3 \sigma$ upper limit of $\sim 4 \times 10^{-14} \mathrm{erg} \mathrm{cm}^{-2} \mathrm{~s}^{-1}$ on the $2-10 \mathrm{keV}$ absorbed flux. For an assumed distance of $7 \mathrm{kpc}$, the luminosity upper limit is $\sim 4 \times 10^{32} \mathrm{erg} \mathrm{s}^{-1}$.

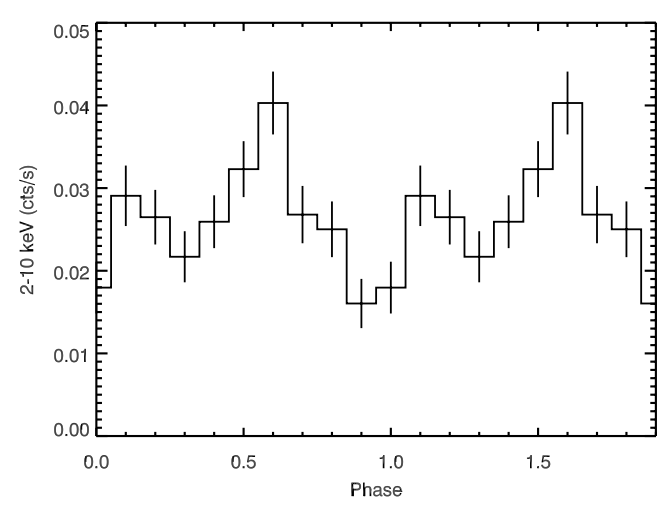

Fig. 1. Light curve of IGR J16358-4726 folded at 5880 s. The light curve is the sum of PN and MOS data and the background has been subtracted. The $\chi^{2}$ statistics corresponds to a probability of $2 \times 10^{-5}$ that the source is constant.

\subsection{Reanalysis of the BeppoSAX observations of SGR 1627-41}

We have reanalyzed the four observations of SGR 1627-41 carried out with the BeppoSAX satellite from August 1998 to September 2000. We extracted the source spectra only from the Medium Energy Concentrator Spectrometer (MECS) data ${ }^{3}$ using circular extraction regions with radius $2^{\prime}$. This relatively small region was chosen in order to reduce the uncertainties related to the background subtraction, since the source lies in a low galactic latitude field affected by the presence of some diffuse emission and other confusing sources. The background spectra were extracted from source free regions of the same observations. The background subtracted spectra in the 1.8-10 keV energy range were rebinned in order to have at least 30 counts per bin and fitted with an absorbed power law. The best fit parameters are reported in Table 1. The small differences with the results reported by Kouveliotou et al. (2003) are probably due to different background estimation techniques.

\section{Discussion}

The light curve of SGR 1627-41 based on data from the different satellites is shown in Fig. 2, where the $2-10 \mathrm{keV}$ flux values correspond to the fits with the same absorption in all the observations $\left(N_{\mathrm{H}}=9 \times 10^{22} \mathrm{~cm}^{-2}\right)$. The long term decrease in luminosity is clear, but, owing to the source spectral variations, the detailed shape of the decay is different for the observed (upper panel) and unabsorbed (lower panel) flux. Kouveliotou et al. (2003) fitted the decay of the unabsorbed flux using a model involving a deep crustal heating following the 1998 bursting activity and requiring a massive neutron $\operatorname{star}\left(M>1.5 M_{\odot}\right)$. In particular, they pointed out that this model could well explain the plateau between days 400 and 800 , but noticed that the March 2003 Chandra observation could not be explained in this framework, suggesting that the source reached a steady low level luminosity. According to our reanalysis of the BeppoSAX data the evidence for a plateau between days 400 and 800 is not

\footnotetext{
3 The Low Energy Concentrator Spectrometer (LECS) data do not add significant information, since SGR 1627-41 was too absorbed and faint for this instrument.
} 


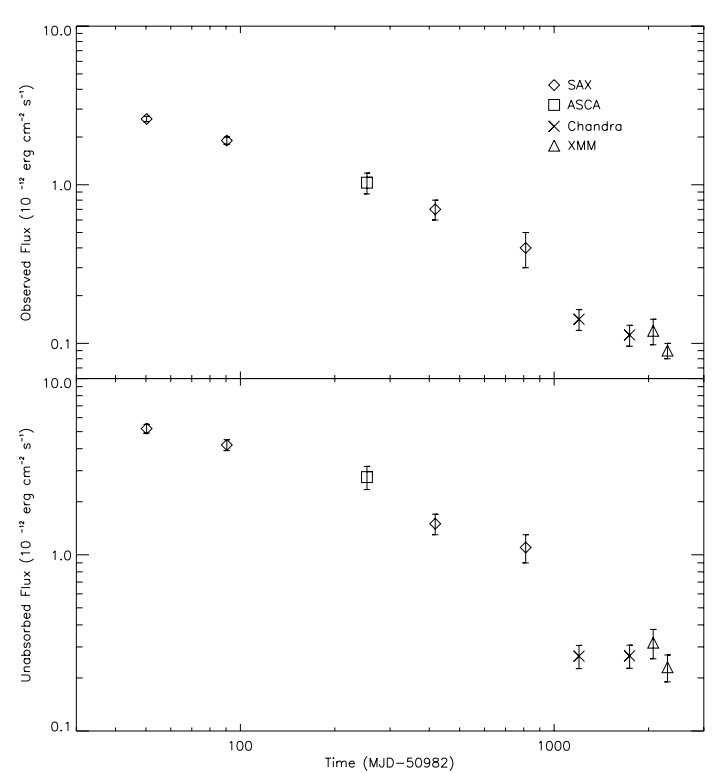

Fig. 2. Long term light curve of SGR 1627-41 based on data from different satellites. Top panel: absorbed flux in the $2-10 \mathrm{keV}$ range. Bottom panel: unabsorbed flux in the $2-10 \mathrm{keV}$ range. The ASCA and Chandra values have been computed from the spectral parameters and unabsorbed fluxes given in Kouveliotou et al. (2003). For clarity, the XMM-Newton points of September 4, which are consistent with the last measurement, are not plotted.

so compelling. In fact all the BeppoSAX and ASCA points, before the rapid decline seen with Chandra in September 2001, are well fit by a power law decay, $F(t) \propto\left(t-t_{0}\right)^{-\delta}$. Fixing $t_{0}$ at the time of the discovery outburst, we obtain $\delta=0.6$.

If one considers the observed fluxes, the Chandra and XMM-Newton data suggest that SGR 1627-41 has continued to fade also after September 2001. There is evidence that the spectrum softened between the two Chandra observations (Kouveliotou et al. 2003). The photon index measured with XMM-Newton is consistent with that of the last Chandra observation but, due to the large uncertainties, also a further softening cannot be excluded. This apparent fading is not necessarily related to a variation of the source overall luminosity, as clearly indicated by the fluxes corrected for the absorption plotted in the lower panel of Fig. 2.

The XMM-Newton data of September 2004 imply a luminosity $\sim 3.5 \times 10^{33} d_{11}^{2} \mathrm{erg} \mathrm{s}^{-1}$. This is the lowest luminosity observed from a SGR. The fact that SGR 1627-41 has not emitted bursts during the last $\sim 6$ years suggests that a luminosity below $10^{34} \mathrm{erg} \mathrm{s}^{-1}$ might be typical of "quiescent" SGRs. This simple interpretation is possibly contradicted by the two following considerations. First, the SGR in the Large Magellanic Cloud, SGR 0526-66, has a higher luminosity $\left(10^{36} \mathrm{erg} \mathrm{s}^{-1}\right)$, but has not shown signs of strong bursting activity in the last 15 years. However, faint bursts, like those recently observed from SGR 1806-20 with INTEGRAL (Götz et al. 2004) might have passed undetected in SGR 0526-66 due to its larger distance and location in a less frequently monitored sky region. Second, most Anomalous X-ray Pulsars (AXPs, see, e.g. Mereghetti et al. 2002, for a review), which are also generally thought to be magnetars, have nearly steady luminosity larger than $10^{35} \mathrm{erg} \mathrm{s}^{-1}$. Although bursts have been observed in three of them (1E 1048.1-5937, Gavriil et al. 2002; 1E 2259+586, Kaspi et al. 2003; XTE J1810-197, Woods et al. 2005), there are a few AXPs which have not shown any bursting activity and yet are relatively luminous $\mathrm{X}$-ray sources.

The low luminosity and soft spectrum of SGR 1627-41 seen with XMM-Newton are quite similar to the values measured in archival data of the AXP XTE J1810-197 (Gotthelf et al. 2004) obtained before its discovery as a bright transient source in January 2003 (Ibrahim et al. 2004). Based on the currently available data, the two sources seem to behave in a similar way. Further observations of XTE J1810-197 will establish if and how a steady quiescent level is attained.

We finally note that another soft repeater, SGR $1900+14$, has possibly been quiescent in the last three years. To our knowledge, the last reported burst activity from this source occurred in November 2002 (Hurley et al. 2002). Therefore it will be interesting to see whether also in SGR $1900+14$ the X-ray luminosity will evolve toward a low state similar to that observed for SGR 1627-41.

Acknowledgements. This work has been partially supported by the Italian Space Agency and by the MIUR under grant PRIN 2004-023189.

\section{References}

Duncan, R. C., \& Thompson, C. 1992, ApJ, 392, L9

Feroci, M., Costa, E., Amati, M., et al. 1998, IAU Circ. No. 6945

Gavriil, F. P., Kaspi, V. M., \& Woods, P. M. 2002, Nature, 419, 142

Gotthelf, E. V., Halpern, J. P., Buxton, M., \& Bailyn, C. 2004, ApJ, 605,368

Götz, D., Mereghetti, S., Mirabel, F. I., \& Hurley, K. 2004a, A\&A, 417, L45

Hurley, K., Kouveliotou, C., Woods, P., et al. 1999, ApJ, 519, L143

Hurley, K., Cline, T., Mazets, E., et al. 2003, GCN Circ., 1715

Ibrahim, A. I., Markwardt, C. B., Swank, J. H., et al. 2004, ApJ, 609, L21

Kaspi, V. M., Gavriil, F. P., Woods, P. M., et al. 2003, ApJ, 588, L93

Kouveliotou, C., Eichler, D., Woods, P. M., et al. 2003, ApJ, 596, L79

Kulkarni, S. R., Kaplan, D. L., Marshall, H. L., et al. 2003, ApJ, 585, 948

Mazets, E. P., Aptekar, R., Butterworth, P., et al. 1999, ApJ, 519, L151

Mereghetti, S., Chiarlone, L., Israel, G. L., \& Stella, L. 2002, in Neutron Stars, Pulsars and Supernova Remnants, ed. W. Becker, H. Lesch, \& J. Trümper, MPE-Report, 278, 29

Mereghetti, S., Tiengo, A., Esposito, P., et al. 2005, ApJ, 628, 938

Patel, S. K., Kouveliotou, C., Tennant, A., et al. 2004, ApJ, 602, L45

Revnivtsev, M. G., Tuerler, M., Del Santo, M., et al. 2003, IAU Circ. N. 8097

Smith, D. A., Bradt, H. V., \& Levine, A. M. 1999, ApJ, 519, L147

Strüder, L., Briel, U., Dennerl, K., et al. 2001, A\&A, 365, L18

Thompson, C., \& Duncan, R. C. 1995, MNRAS, 275, 255

Turner, M. J. L., Abbey, A., Arnaud, M., et al. 2001, A\&A, 365, L27

Wachter, S., Patel, S. K., Kouveliotou, C., et al. 2004, ApJ, 615, 887

Woods, P. M., \& Thompson, C. 2004 [arXiv: astro-ph/0406133]

Woods, P. M., Kouveliotou, C., van Paradijs, J., et al. 1999, ApJ, 519, L139

Woods, P. M., Kouveliotou, C., Gögüs, E., et al. 2001, ApJ, 552, 748

Woods, P. M., Kouveliotou, C., Gavriil, F. P., et al. 2005, ApJ, 629, 985 